\title{
Die Verteilung der Steuer- und Abgabenlast
}

\author{
Im Rahmen einer Querschnittsanalyse wird die Steuer- und Abgabenlast in Deutschland auf \\ Haushaltsebene für 2018 untersucht. Neben der Belastung nach Haushaltseinkommen wird \\ die Belastung nach der Konsumhöhe betrachtet. Schließlich wird der Beitrag unterschiedlicher \\ Einkommensgruppen zur Staatsfinanzierung dargestellt. Es zeigt sich, dass die \\ durchschnittliche Abgabenquote $40 \%$ bei einem Bruttoeinkommen von 24.000 Euro beträgt. \\ Bei einem Einkommen von 80.000 Euro steigt die Quote auf über $50 \%$.
}

\begin{abstract}
In Relation zur Wirtschaftsleistung ist die Abgabenlast in den 1990er Jahren gestiegen, in den 2000er Jahren zurückgegangen und in den 2010er Jahren wieder merklich angewachsen. Mittlerweile befindet sich die Abgabenlast nahe dem Stand von 1991 (Statistisches Bundesamt, 2021). Dieser Beitrag untersucht, wie sich die Steuer- und Abgabenlast in Deutschland $2018^{1}$ auf die privaten Haushalte verteilte. Hierzu werden zwei repräsentative Stichproben deutscher Haushalte kombiniert: das Sozio-oekonomische Panel (SOEP) und die Einkommens- und Verbrauchsstichprobe (EVS). Die Steuer- und Abgabenlast jedes Haushalts wird mithilfe des Mikrosimulationsmodells des RWI berechnet.
\end{abstract}

Die Simulationen zeigen, dass die durchschnittliche Abgabenlast in Relation zum Bruttoeinkommen schon bei einem jährlichen Bruttoeinkommen von 24.000 Euro $40 \%$ übersteigt. Bei einem Einkommen von 35.000 Euro übersteigt die durchschnittliche Abgabenlast $45 \%$ und bei einem Einkommen von 80.000 Euro $50 \%$. Beschränkt man die Analyse auf abhängig oder selbstständig Beschäftigte, werden diese Abgabenquoten bei deutlich geringeren Jahreseinkommen erreicht. Am unteren Ende der Ein-

(C) Der/die Autor:in(nen) 2021. Open Access: Dieser Artikel wird unter der Creative Commons Namensnennung 4.0 International Lizenz veröffentlicht (creativecommons.org/licenses/by/4.0/deed.de).

Open Access wird durch die ZBW - Leibniz-Informationszentrum Wirtschaft gefördert.

* Der Artikel basiert auf einem Gutachten, das im Auftrag der FriedrichNaumann-Stiftung für die Freiheit erarbeitet wurde.

1 Das aktuellste Jahr, für das Daten der EVS vorhanden sind. Die verwendete Welle des SOEPs bezieht sich auf das Jahr 2017 und wurde um ein Jahr fortgeschrieben.

Niklas Isaak, Dr. Philipp Jäger und Dr. Robin Jessen sind Wissenschaftler im Kompetenzbereich Wachstum, Konjunktur, Öffentliche Finanzen am RWI - Leibniz-Institut für Wirtschaftsforschung. kommensverteilung liegt die durchschnittliche Abgabenlast bei über $50 \%$, fällt dann zunächst auf unter $30 \%$ und steigt anschließend rapide an. Bei höheren Einkommen steigt sie nur noch langsam. Der höchste Wert wird bei Jahreseinkommen von rund 100.000 Euro erreicht. Ab hier sinkt die durchschnittliche Abgabenquote allmählich, weil Sozialversicherungsbeiträge weniger stark ins Gewicht fallen.

Zur Einordnung der Ergebnisse seien vier Punkte angemerkt. Erstens wird eine Reihe von Abgaben nicht erfasst. So wird beispielsweise die Erbschaftsteuer aufgrund fehlender Daten nicht berücksichtigt. Zweitens sind sehr hohe Einkommen in den verwendeten Datensätzen untererfasst. Dies dürfte sich allerdings auf das oberste Perzentil beschränken (vgl. Bartels und Metzing, 2019). Drittens hängt die Verteilungs- und Belastungswirkung des Abgabensystems nicht nur unmittelbar von den berechneten Abgabenquoten, sondern auch von Verhaltensanpassungen der Individuen ab. Wenn z.B. das Einkommensteuersystem dazu führt, dass Personen weniger arbeiten, drückt sich darin die Zusatzlast der verzerrenden Besteuerung aus, die in der vorliegenden Analyse nicht erfasst ist. Viertens betrachtet die Studie die Abgabenlast im Querschnitt, d.h., es wird nur ein Jahr und nicht der gesamte Lebenszyklus der Individuen betrachtet, was bei der Interpretation zu berücksichtigen ist. So erweckt die hohe durchschnittliche Abgabenquote am unteren Ende der Einkommensverteilung den Eindruck, dass das Abgabensystem niedrige Einkommen höher belaste. Der Effekt ergibt sich teilweise aus der Betrachtung nur eines Jahres. Haushalte, die in einem Jahr über ein niedriges Einkommen verfügen, wie Studierende oder Arbeitslose, konsumieren durchschnittlich in diesem Jahr einen hohen Teil des Einkommens oder bauen sogar Vermögen ab. Betrachtet man die Abgabenquote relativ zur Höhe des jährlichen Konsums anstatt in Relation zum Einkommen, ist das lokale Maximum bei niedrigen Einkommen deutlich abgeschwächt.

Die vorliegende Studie ergänzt eine Reihe von Studien über die Steuer- und Abgabenlast in Deutschland (Bach et al., 2016; RWI, 2011, 2015, 2017; RWI und FiFo Köln, 2007, 
2009). Bach et al. (2016) untersuchen die Verteilungswirkung des Steuer- und Transfersystems unter Verwendung eines integrierten Datensatzes auf Basis verschiedener Mikrodatensätze für 2015. Die Ergebnisse sind in Teilen mit denen der vorliegenden Studie vergleichbar. ${ }^{2}$ Verwandte Literatur analysiert die Entwicklung der Einkommensverteilung unter Berücksichtigung des Abgaben- und Transfersystems (z. B. Biewen und Juhasz, 2012; Bach et al., 2009; Biewen et al., 2019; Jessen, 2019) oder berechnet die Progressivität des Umverteilungssystems mithilfe von Kennzahlen (z. B. Bach et al., 2016; Schaefer und Peichl, 2008).

\section{Verwendete Daten und Simulationsmodell}

Die Analyse erfolgt auf Grundlage von zwei Haushaltsumfragen: dem Sozio-oekonomischen Panel (SOEP, vgl. Goebel et al., 2019) sowie der Einkommens- und Verbrauchsstichprobe (EVS). Während das SOEP zur Simulation der Einkommensteuern, Sozialbeiträge und Transfers verwendet wird, nutzen wir die EVS für die Analyse der indirekten Steuern (Umsatzsteuer, Energiesteuer etc.).

Das SOEP ist eine für Deutschland repräsentative Haushaltsbefragung. Es enthält Informationen zu etwa 20.000 Privathaushalten. Die Teilnahme an der Befragung ist freiwillig. Die befragten Haushalte und Individuen geben ausführlich Auskunft über ihre privaten Lebensbedingungen und Einkommensverhältnisse, wie z. B. die Höhe und Quellen des Einkommens und den Transferbezug. Wir verwenden die Welle v35, die retrospektive Informationen über 2017 enthält. Arbeitseinkommen werden mit der Wachstumsrate der Bruttolöhne und -gehälter in 2018 fortgeschrieben, andere Einkommen mit der Inflationsrate.

Bei der EVS handelt es sich um eine Quotenstichprobe, die vom Statistischen Bundesamt in Zusammenarbeit mit den Landesämtern alle fünf Jahre durchgeführt wird. An der repräsentativen Befragung nehmen rund 60.000 Haushalte teil. Die Teilnahme an der Befragung ist freiwillig. Da der Datensatz im Vergleich zum SOEP sehr detaillierte Angaben zu den Ausgaben der befragten Haushalte enthält, ist er besser geeignet, die Belastung durch Verbrauchsteuern zu ermitteln. Die EVS erfasst allerdings keine Haushalte mit einem monatlichen Haushaltsnettoeinkommen über 18.000 Euro. Wir verwenden die Welle 2018.

Zur Berechnung der Steuer- und Abgabenlast nutzen wir das Mikrosimulationsmodell des RWI (RWI-Einkommensteuer-Mikrosimulationsmodell, EMSIM, vgl. Bechara et

2 Eine alternative Herangehensweise ist die der „Distributional National Accounts“ (Piketty et al., 2018). Hierbei werden Mikrodatensätze so angepasst, dass die aus den Mikrodaten berechneten Aggregate denen der VGR entsprechen. al., 2015). Das EMSIM bildet das deutsche Steuer-, Transfer- und Sozialabgabensystem umfangreich ab und basiert auf den Haushaltsdaten des SOEP. Es berechnet für jeden Haushalt dieser Befragung auf Basis der soziodemografischen Charakteristika und des Markteinkommens der einzelnen Mitglieder die verfügbaren Einkommen sowie gegebenenfalls die bezogenen Transfers auf Basis der tatsächlichen Rechtslage von 2018. Mithilfe des EMSIM kann für die vorliegende Untersuchung insbesondere die Belastung durch Einkommensteuer und Sozialversicherungsbeiträge detailliert simuliert werden.

Die Belastung durch konsumbezogene Steuern wird mithilfe der EVS-Daten hinzugeschätzt. Die Ergebnisse werden entlang der Einkommensverteilung des SOEP dargestellt. Um die auf Basis der EVS berechnete Belastung durch Verbrauchsteuern auf das SOEP zu übertragen, wird zunächst in der EVS der Zusammenhang zwischen Haushaltscharakteristika und Steuerschuld sowie Konsumlevel geschätzt. Dies geschieht mittels einer Regression dieser abhängigen Variablen auf binäre Variablen für das Alter der Haushaltsmitglieder, Nettoeinkommen und die Haushaltszusammensetzung. Die geschätzten Parameter werden in einem zweiten Schritt verwendet, um die Höhe des Konsums und der Verbrauchsteuern im SOEP zu imputieren.

Für die Belastung durch Unternehmensteuern wird angenommen, dass die Unternehmensteuern sowohl Löhne als auch Kapitaleinkünfte senken. Dazu unterstellen wir, dass die Summe der Aggregate von Löhnen und Kapitaleinkommen um den Betrag des Aggregats der Unternehmensteuern höher wäre, wenn es keine gäbe. Die Belastung der Haushalte durch Unternehmensteuern ergibt sich dann aus der Differenz zwischen den kontrafaktischen und den tatsächlichen Löhnen bzw. Kapitaleinkünften.

\section{Einkommenskonzepte und Abgabenblöcke}

Die Bezugsgröße der Belastungsanalyse bildet das Haushaltsbruttoeinkommen, das sich aus der Summe der Bruttoeinkommen aller Haushaltsmitglieder zusammensetzt. Das Bruttoeinkommen enthält Markteinkommen aus selbstständiger und nichtselbstständiger Tätigkeit, Immobilienbesitz (Mieten) oder Kapitalanlagen. Dazu kommen gesetzliche und private Transfers (z. B. Lohnersatzleistungen der Sozialversicherung, Kindergeld, Sozialleistungen) sowie Renten und Pensionen und andere staatliche Transfers. Wir beschränken uns auf ein enges Einkommenskonzept. Fiktive Einkommen, z.B. aus selbstgenutzten Vermögenswerten, bilden keinen Teil des Bruttoeinkommens. Wir berücksichtigen allerdings, dass die tatsächlichen Einkommenszuflüsse häufig nicht den Markteinkommen entsprechen. So sind die empfangenen Bruttolöhne nicht mit dem Markteinkommen aus nichtselbstständiger Arbeit 
identisch, da die Löhne bereits um Arbeitgeberbeiträge zur Sozialversicherung und Unternehmensteuern gemindert wurden. Um Haushalte mit unterschiedlicher Personenzahl und Altersstruktur vergleichen zu können, berechnen wir für jeden Haushalt das Haushaltsbruttoäquivalenzeinkommen. Dazu teilen wir das Haushaltsbruttoeinkommen durch die bedarfsgewichtete Zahl der Haushaltsmitglieder. ${ }^{3}$ Nur so kann berücksichtigt werden, dass Haushalte je nach Konstellation zum Erreichen des gleichen Lebensstandards unterschiedlich hohe Pro-Kopf-Einkommen benötigen.

Die Analyse berücksichtigt vier Abgabenblöcke:

1. Einkommensteuer und Solidaritätszuschlag werden anhand des simulierten zu versteuernden Einkommens basierend auf dem Einkommensteuertarif sowie dem Tarif der Kapitalertragsteuer von 2018 berechnet.

2. Sozialversicherungsbeiträge beinhalten Arbeitnehmerund Arbeitgeberbeiträge zur gesetzlichen Renten-, Kranken-, Arbeitslosen-, Pflege- und Unfallversicherung. Sie berechnen sich als Produkt aus dem beitragspflichtigen Teil des Bruttolohns und dem gesetzlich festgelegten (Renten-, Arbeitslosen- und Pflegeversicherung) oder durchschnittlichen Beitragssatz (Krankenversicherung, Unfallversicherung). Beiträge zur privaten Krankenversicherung werden ebenfalls berücksichtigt aber separat ausgewiesen, da es gewisse Wahlmöglichkeiten bezüglich des Umfangs des Versicherungsschutzes gibt. Nicht erfasst werden unterstellte Sozialbeiträge des Staats.

3. Verbrauchsteuern beinhalten die Umsatzsteuer, Energie- und Stromsteuer, die EEG-Umlage sowie Steuern auf Alkohol und Tabakprodukte. Die Steuerlast wird basierend auf Durchschnittspreisen und tatsächlichen Ausgaben der Haushalte sowie den Steuersätzen von 2018 berechnet. Nicht berücksichtigt wird die Auswirkung unterschiedlicher Steuersätze von Vorleistungsgütern auf die Endverbraucherpreise.

4. Die erfassten Unternehmensteuern beinhalten die Körperschaftsteuer, Gewerbesteuer und Kapitalertragsteuer von Kapitalgesellschaften. Gewerbesteuerzahlungen von Einzelunternehmen und Personengesellschaften werden nicht explizit berücksichtigt, da sie in der Regel nahezu vollständig von der Einkommensteuer abziehbar sind. In der Simulation wird die Gewerbesteuer von Ein-

3 Wir nutzen dazu die modifizierte OECD-Skala. Dabei erhält der erste Erwachsene im Haushalt ein Bedarfsgewicht von 1, jeder weitere Erwachsene ein Gewicht von 0,5 und Kinder bis 14 Jahren ein Gewicht von 0,3. Z. B. wird das Bruttoeinkommen eines Haushalts mit zwei Erwachsenen und einem Kind durch 1+0,5+0,3=1,8 geteilt.
Abbildung 1

Die absolute Abgabenlast nach Einkommen

in Euro

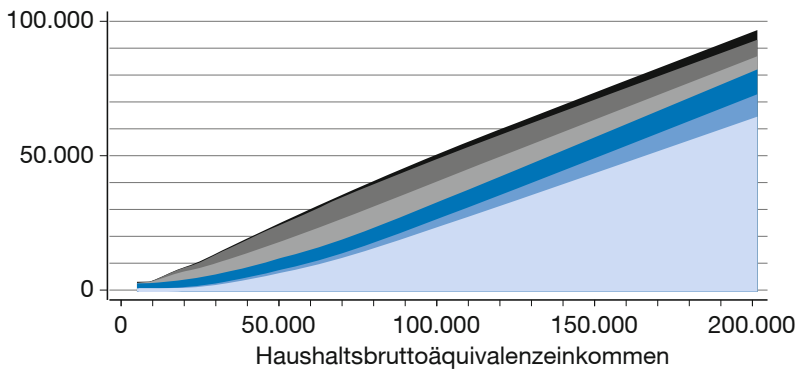

Private Krankenversicherung Arbeitgeberbeiträge Sozialbeiträge Haushalte

Unternehmensteuern Indirekte Steuern

Einkommensteuern Haushalte

Quelle: eigene Berechnung auf Grundlage von SOEP v35 und EVS (2018). Rechtsstand 2018. Mit lokaler Regression geglättet.

zelunternehmen und Personengesellschaften der Einkommensteuer zugeordnet.

Nicht berücksichtigt werden Gebühren, Grundsteuern, Erbschaftsteuer und der Rundfunkbeitrag. Auch Verbrauchsteuern auf Güter, die nicht unmittelbar von Haushalten nachgefragt werden, bleiben unberücksichtigt.

\section{Inzidenzannahmen}

Wir treffen folgende Inzidenzannahmen im Einklang mit der Literatur (z. B. Bach et al., 2016; RWI, 2017). Wir gehen implizit davon aus, dass es keine Verhaltensanpassungen gibt und Personen somit auf eine veränderte Abgabenlast nicht durch eine Änderung ihres Arbeitsangebots reagieren. Die Haushalte tragen Einkommensteuer und Sozialversicherungsbeiträge (Arbeitnehmer- und Arbeitgeberbeiträge) sowie Verbrauchsteuern. Die Unternehmensteuern gehen jeweils hälftig zulasten der Unternehmensgewinne und Arbeitnehmerlöhne. ${ }^{4}$ Wir nehmen an, dass $50 \%$ der Unternehmensteuern die Kapitalerträge der privaten Haushalte mindern. Im Modell werden die Unternehmensteuern dazu proportional zu den tatsächlich bezogenen Kapitalerträgen verteilt. Die anderen $50 \%$ der Unternehmensgewinne reduzieren die Arbeitnehmerlöhne. Wir unterstellen, dass die Löhne aller Arbeitnehmer aufgrund der Unternehmensteuer um den gleichen Prozentsatz gemindert wurden. Dafür spricht die jüngere empirische Evidenz für Deutschland, die darauf hindeutet, dass Unternehmensteuern etwa hälftig die Lohneinkommen belasten (Fuest et al., 2018; Dwenger et al., 2019).

4 In einer längeren Version dieser Studie wurde zudem ein Alternativszenario berechnet, in dem die Unternehmensteuern ausschließlich zulasten der Kapitalerträge gehen. Dies führt zu einer etwas stärkeren Belastung der höheren Einkommen. 
Abbildung 2

Die relative Abgabenlast nach Einkommen

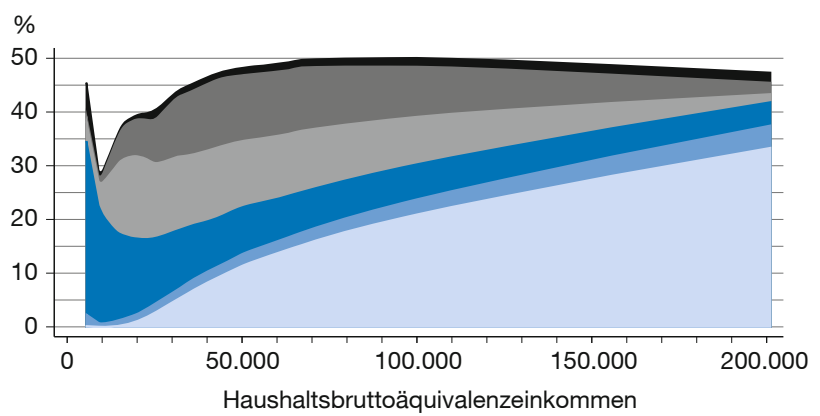

Private Krankenversicherung Sozialbeiträge Haushalte Unternehmensteuern
Abbildung 3

Die relative Abgabenlast nach Einkommensverteilung

$$
\%
$$

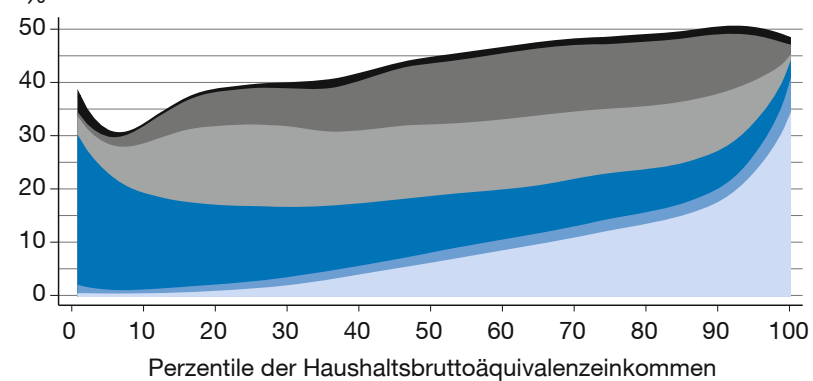

Perzentile der Haushaltsbruttoäquivalenzeinkommen

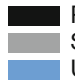

Private Krankenversicherung Sozialbeiträge Haushalte Unternehmensteuern
Quelle: eigene Berechnung auf Grundlage des SOEP v35 und der EVS (2018). Rechtsstand 2018. Mit lokaler Regression geglättet.

\section{Ergebnisse}

\section{Belastung der Haushalte nach Einkommen}

Abbildung 1 zeigt die durchschnittliche absolute jährliche Belastung deutscher Haushalte 2018 mit Steuern und weiteren Abgaben in Abhängigkeit ihres Haushaltseinkommens. Die Abbildung verdeutlicht, dass die absolute Abgabenlast stetig mit dem Einkommen steigt. So fallen bei einem Haushaltsbruttoäquivalenzeinkommen von 20.000 Euro im Durchschnitt Steuern und Abgaben in Höhe von rund 8.000 Euro an. Bei Haushaltsbruttoäquivalenzeinkommen von 50.000 Euro beträgt die Abgabenlast rund 24.000 Euro, bei 100.000 Euro rund 50.000 Euro. Die relative Bedeutung unterschiedlicher Steuern und Abgaben unterscheidet sich je nach Einkommenshöhe deutlich. Während im unteren Einkommensbereich indirekte Steuern und Sozialversicherungsbeiträge den größten Teil der Belastung ausmachen, werden einkommensstärkere Haushalte vor allem durch Einkommensteuern belastet.

Setzt man die Abgabenlast ins Verhältnis zum Einkommen (vgl. Abbildung 2), wird deutlich, dass die Abgabenquote stark vom Haushaltseinkommen abhängt. Haushalte mit niedrigen Haushaltseinkommen müssen im Mittel einen geringeren Teil ihres Einkommens abgeben als einkommensstärkere Haushalte. ${ }^{5}$ Dabei steigt die Abgabenquote mit steigenden Einkommen zunächst kräftig an. Während

5 Bei sehr niedrigen Einkommen liegt die Abgabenquote relativ hoch. Dies ist auf die hohe Konsumquote dieser Haushalte zurückzuführen. Wird sogar Vermögen abgebaut, etwa bei Rentner:innen, können die Konsumausgaben höher als die Einkommen ausfallen. Gemessen am Einkommen liegen die konsumbezogenen Steuern für diese Haushalte dadurch sehr hoch. Teils wird dies als Beleg dafür angeführt, dass Verbrauchsteuern regressiv seien. Diese Argumentation ist schon deshalb falsch, weil sich der Progressionsgrad einer Steuer in Bezug auf die jeweilige Steuerbasis bemisst.
Quelle: eigene Berechnung auf Grundlage des SOEP v35 und der EVS (2018). Rechtsstand 2018. Mit lokaler Regression geglättet.

die Abgabenquote bei einem Haushaltsbruttoäquivalenzeinkommen von 10.000 Euro rund $30 \%$ beträgt, liegt sie bei Einkommen ab knapp 24.000 Euro bereits oberhalb von $40 \%$. Bei einem Einkommen von 35.000 Euro beträgt die Abgabenquote $45 \%$. Ab einem Haushaltsbruttoäquivalenzeinkommen von rund 70.000 Euro flacht der Anstieg der Abgabenquote ab, bis die Spitzenabgabenlast bei einem Äquivalenzeinkommen von rund 100.000 Euro erreicht wird. In diesem Einkommensbereich beträgt die Abgabenquote über $50 \%$. Danach sinkt die Abgabenquote mit steigendem Einkommen leicht, aber stetig. Der Rückgang der Abgabenquote im obersten Einkommenssegment ist auf im Verhältnis zum Bruttoeinkommen relativ geringere Sozialversicherungsbeiträge (aufgrund der Beitragsbemessungsgrenze) und niedrigere indirekte Steuern (durch höhere Sparquoten) zurückzuführen, die nicht komplett durch höhere Einkommen- und Unternehmensteuern aufgewogen werden. Abbildung 3 zeigt die Abgabenquote nach Dezilen der Einkommensverteilung. Ab dem 34. Einkommensperzentil liegt die durchschnittliche Abgabenquote über $40 \%$. In der Mitte der Einkommensverteilung liegt die Abgabenquote bei rund $45 \%$. Etwa $7 \%$ der Haushalte zwischen dem 89. und 95. Perzentil, geben mehr als die Hälfte ihres Einkommens als Abgaben ab. Im Vergleich zur gesamten Bevölkerung sind die Abgabenquoten für Berufstätige deutlich höher (vgl. Abbildung 4). Ein Grund dafür ist, dass Renten nur anteilig der Einkommensteuer unterliegen. Eine durchschnittliche Abgabenquote von $40 \%$ wird bei Berufstätigen schon ab einem Einkommen von 17.000 Euro erreicht. Ab einem Einkommen von 54.000 Euro geben Berufstätige im Durchschnitt gar die Hälfte ihres Einkommens ab.

Bislang haben wir nur die Einnahmenseite des Staats und die damit einhergehende Abgabenlast betrachtet. Bei vielen Haushalten stehen den Abgaben allerdings staatliche 


\section{Abbildung 4}

Die relative Abgabenlast nach Einkommen: Berufstätige $\%$

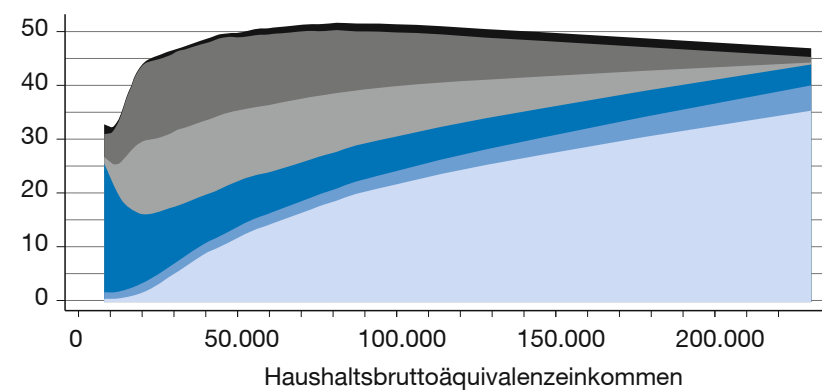

Haushaltsbruttoäquivalenzeinkommen

Private Krankenversicherung Sozialbeiträge Haushalte Unternehmensteuern

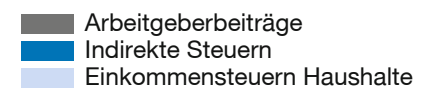

Quelle: eigene Berechnung auf Grundlage des SOEP v35 und der EVS (2018). Rechtsstand 2018. Mit lokaler Regression geglättet.

\section{Abbildung 5}

Die relative Abgabenlast und Transfers entlang der Einkommensverteilung

$\%$

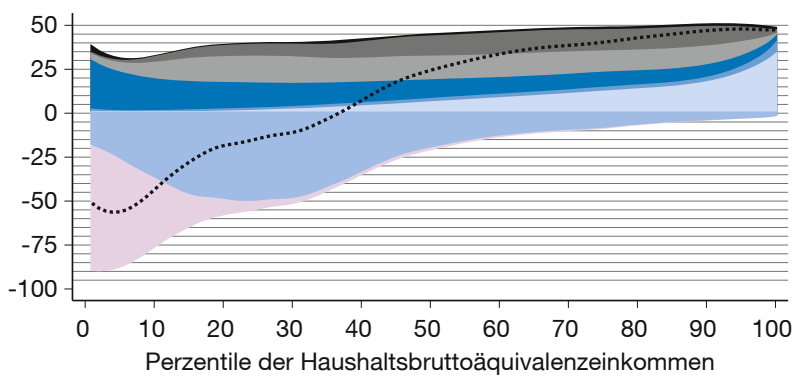

Private Krankenversicherung Sozialbeiträge Haushalte

Unternehmensteuern

Sonstige Transfers

...... Nettobelastung

Quelle: eigene Berechnung auf Grundlage des SOEP v35 und der EVS (2018). Rechtsstand 2018. Mit lokaler Regression geglättet.

\section{Abbildung 6}

Die Abgabenlast relativ zum Bruttokonsum entlang der Konsumverteilung

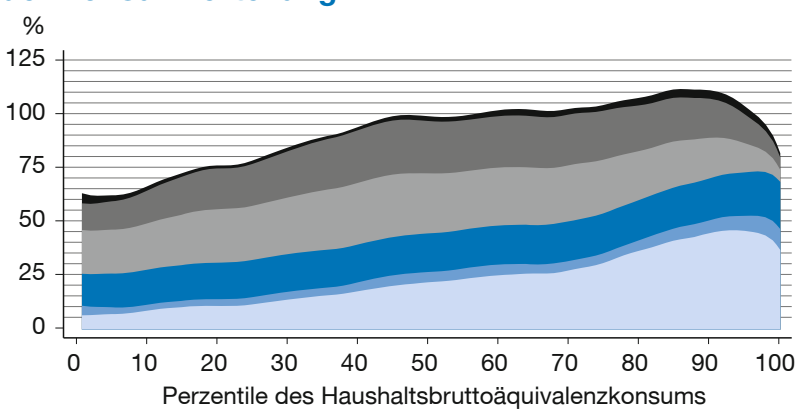

Private Krankenversicherung Sozialbeiträge Haushalte

Unternehmensteuern

Arbeitgeberbeiträge Indirekte Steuern

Einkommensteuern Haushalte

Quelle: eigene Berechnung auf Grundlage des SOEP v35 und der EVS (2018). Rechtsstand 2018. Mit lokaler Regression geglättet.
Transfers (Grundsicherung, Sozialleistungen, Renten) entgegen. Die Nettoabgabenlast - gezahlte Steuern und Abgaben abzüglich empfangener Geldleistungen des Staats - liegt daher für viele Haushalte unterhalb der bisher ausgewiesenen (Brutto-)Abgabenlast. Gemessen an der Einkommensverteilung (vgl. Abbildung 5) wird deutlich, dass ein Großteil der Haushalte staatliche Transfers erhält. So sind die unteren $36 \%$ der Einkommensverteilung Nettoempfänger staatlicher Geldleistungen. Bei sehr niedrigen Einkommen sind die Transfers stark durch Arbeitslosengeld und Arbeitslosengeld II geprägt. Bei etwas höheren Einkommen nehmen Altersrenten eine stärke Rolle ein. Auch die Nettobelastung für Haushalte in der Mitte der Einkommensverteilung fällt mit rund $24 \%$ deutlich niedriger aus als die Abgabenquote (45\%). Da die Transfers im sehr hohen Einkommenssegment kaum noch eine Rolle spielen, unterscheidet sich die Nettobelastungsquote oberhalb des 90. Perzentils kaum noch von der Abgabenquote.

\section{Die Steuer- und Abgabenlast nach Konsumhöhe}

Nun wird die Abgabenlast nicht entlang des Einkommens, sondern entlang des Konsums betrachtet. ${ }^{6}$ Hintergrund ist die Überlegung, dass auch gesparte Einkommen irgendwann verkonsumiert werden und in diesem Moment auch Konsumsteuern anfallen. Ein ausschließlicher Fokus auf die Einkommen könnte verzerrend wirken, da unterstellt wird, dass auf gesparte Einkommen keine Konsumsteuern anfallen. Dies gilt insbesondere in der Querschnittsbetrachtung, in der nur ein Jahr des Lebenszyklus dargestellt wird. Abbildung 6 zeigt allerdings, dass sich die Ergebnisse zwischen Einkommens- und Konsumbetrachtung qualitativ ähneln. Unterschiede ergeben sich durch die unterschiedliche Skalierung. Die relative Belastung fällt deutlich höher aus, da die absolute Abgabenlast nun ins Verhältnis zum Haushaltskonsum gesetzt wird. Dadurch ist es möglich, dass die Abgabenquote 100\% überschreitet. Sie besagt in diesem Fall, dass die absolute Abgabenlast größer ist als der Haushaltskonsum. Ähnlich wie bei der Einkommensbetrachtung fällt die höchste Belastung dabei nahe des 90 . Perzentils an. Während bei der Betrachtung nach Einkommen die Belastung am unteren Rand verhältnismäßig hoch ist, weil das Konsumniveau der Niedrigverdienenden durchschnittlich im Verhältnis zum verfügbaren Einkommen hoch ist, ist dieser Effekt bei der Betrachtung nach Konsum deutlich weniger

6 Für diesen Abschnitt wird die Imputationsmethode angepasst. Nachdem die Konsumlevel und Verbrauchsteuerbelastung auf Haushaltsebene im SOEP auf Basis der in der EVS geschätzten Parameter imputiert wurde, werden, gesondert nach Einkommens-, Alters- und Haushaltstypgruppen, die Residuen der jeweiligen Schätzung in der EVS gezogen und an das SOEP herangespielt. Damit entspricht für eine bestimmte Haushaltsgruppe nicht nur das durchschnittliche Konsumniveau, sondern auch die Varianz des im SOEP imputierten Konsums dem der EVS. 


\section{Abbildung 7}

\section{Die Konzentrationskurve der Abgabenlast}

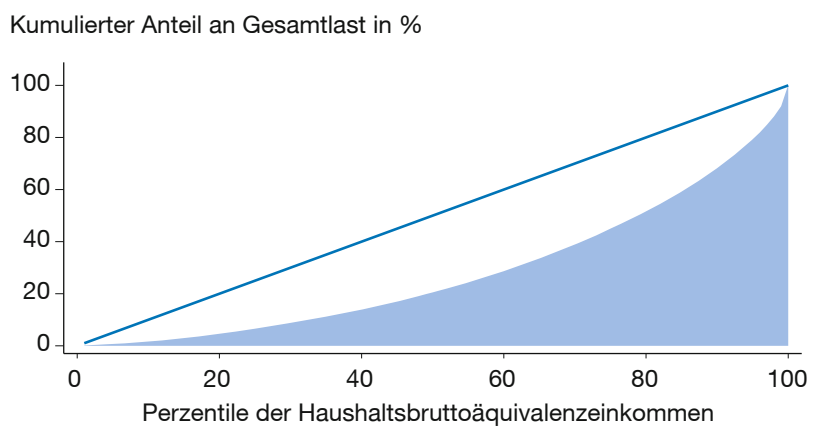

Quelle: eigene Berechnung auf Grundlage von SOEP und EVS (2018).

stark ausgeprägt. Mit sehr hohen Konsumniveaus nimmt die relative Durchschnittsbelastung ab, da im Mittel bei Haushalten mit hohem Konsumniveau die Konsumquote hoch ist. Daher spielen Einkommensteuern für Haushalte mit hohem Konsumniveau eine relativ geringere Rolle.

\section{Beitrag der Einkommensgruppen zur Staatsfinanzierung}

Neben der absoluten und relativen Belastung stellt sich die Frage, welchen Beitrag unterschiedliche Einkommensgruppen zur Staatsfinanzierung leisten. Abbildung 7 stellt mittels einer Konzentrationskurve dar, welchen kumulativen Anteil einzelne Einkommensgruppen an der gesamten Abgabenlast tragen. Die Darstellung beruht auf denselben Berechnungen wie die vorherigen Abschnitte, so sind dieselben Steuern und Abgaben erfasst, einzig die Beiträge zur privaten Krankenversicherung sind hier nicht berücksichtigt. Die gerade Linie zeigt die hypothetische Situation, in der jedes Perzentil der Einkommensverteilung den gleichen Anteil der Gesamtlast trägt. Dies wäre der Fall, wenn ausschließlich Kopfsteuern verwendet würden. Je größer die Fläche zwischen der geraden Linie und der blauen Fläche, desto höher ist der Beitrag der Haushalte mit hohem Einkommen an der Staatsfinanzierung. Während die untere Hälfte der Einkommensverteilung rund $20 \%$ der gesamten Abgabenlast trägt, entfallen auf die obere Hälfte die verbliebenen $80 \%$. Dabei gilt, je höher die Einkommen, desto stärker steigt der Anteil an der Gesamtlast. So entfallen auf die oberen $20 \%$ rund $50 \%$ der Abgabenlast und auf die Top
$10 \%$ etwa $30 \%$. Da die Topeinkommensbezieher:innen im SOEP unterrepräsentiert sind, dürfte der tatsächliche Anteil an der Gesamtabgabenlast speziell im oberen $1 \%$ der Einkommensverteilung noch deutlich höher ausfallen.

\section{Literatur}

Bach, S., M. Beznoska und V. Steiner (2016), Aufkommens- und Verteilungswirkungen einer Wiedererhebung der Vermögensteuer in Deutschland, DIW Politikberatung Kompakt, 108.

Bach, S., G. Corneo und V. Steiner (2009), From Bottom to Top: The Entire Income Distribution in Germany, 1992-2003, Review of Income and Wealth, 55, 331-359.

Bartels, C. und M. Metzing (2019), An Integrated Approach for a Top-corrected Income Distribution, Journal of Economic Inequality, 17(2), 125-143.

Bechara, P., T. Kasten und S. Schaffner (2015), Dokumentation des RWI-Einkommensteuer-Mikrosimulationsmodells (EMSIM), RWI-Materialien, 86.

Biewen, M. und A. Juhasz (2012), Understanding Rising Income Inequality in Germany, 1999/2000-2005/2006, Review of Income and Wealth, 58(4), 622-647.

Biewen, M., M. Ungerer und M. Löffler (2019), Why did income inequality in Germany not increase further after 2005?, German Economic Review, 20(4), 471-504.

Dwenger, N., P. Rattenhuber und V. Steiner (2019), Sharing the Burden? Empirical Evidence on Corporate Tax Incidence, German Economic Review, 20(4), 107-140.

Fuest, C., A. Peichl und S. Siegloch (2018), Do Higher Corporate Taxes Reduce Wages? Micro Evidence from Germany, American Economic Review, 108(2), 393-418.

Goebel, J., M. M. Grabka, S. Liebig, M. Kroh, D. Richter, C. Schröder und J. Schupp (2019), The German Socio-Economic Panel (SOEP), Journal of Economics and Statistics, 239(2), 345-360.

Jessen, R. (2019), Why has Income Inequality in Germany Increased from 2002 to 2011? A Behavioral Microsimulation Decomposition, Review of Income and Wealth, 65(3), 540-560.

RWI (2011), Wer trägt den Staat? Eine Analyse von Steuer- und Abgabenlasten, RWI Position, 43.

RWI (2015), Wer trägt den Staat im Jahr 2015? Die aktuelle Verteilung der Steuer- und Abgabenlasten auf die Bevölkerung in Deutschland, RWI Projektberichte.

RWI (2017), Steuer- und Abgabenlast in Deutschland - Eine Analyse auf Makro- und Mikroebene. Endbericht. Gutachten im Auftrag der Friedrich-Naumann-Stiftung für die Freiheit, RWI Projektberichte.

RWI und FiFo Köln (2007), Der Zusammenhang zwischen Steuerlast- und Einkommensverteilung, RWI Projektberichte.

RWI und FiFo Köln (2009), Wer trägt den Staat? Die aktuelle Verteilung von Steuer- und Beitragslasten auf die Bevölkerung in Deutschland, RWI Projektberichte.

Piketty, T., E. Saez und G. Zucman (2018), Distributional National Accounts: Methods and Estimates for the United States, The Quarterly Journal of Economics, 133(2), 553-609.

Schaefer, T. und A. Peichl (2008), Wie progressiv ist Deutschland?, FiFo Discussion Papers - Finanzwissenschaftliche Diskussionsbeiträge, 08(5), Universität Köln, FiFo Institute for Public Economics.

Statistisches Bundesamt (2021), Volkswirtschaftliche Gesamtrechnungen - Inlandsproduktberechnung, Vierteljahresergebnisse, Fachserie 18 Reihe 1.2. Wiesbaden.

Title: The Distribution of the Tax and Social Security Burden

Abstract: Using a cross-sectional analysis, this article investigates the burden of taxation and social security contributions across German households. The burden is determined with respect to household income as well as consumption level. Moreover, the different income groups' contributions to public finance are illustrated. It can be demonstrated that for gross incomes of 24,000 euros, the average tax and contribution ratio amounts to $40 \%$. For household incomes of 80,000 euros, the ratio rises above $50 \%$.

JEL Classification: H2O, H22, H24 